EPJ Web of Conferences 16, 05005 (2011)

DOI: $10.1051 /$ epjconf/20111605005

(C) Owned by the authors, published by EDP Sciences, 2011

\title{
Eccentricity formation and habitable planets in OGLE-06-109L system
}

\author{
S. Wang ${ }^{\text {a }}$ and J.-L. Zhou \\ Department of Astronomy, Nanjing University, Nanjing 210093, China
}

\begin{abstract}
Recent observation of microlensing technique reveals two giant planets at 2.3 AU and 4.6 AU around the star OGLE-06-109L. We investigate the eccentricity formation of these two planets, and find that planetary scattering or resonance crossing may be the reason for their eccentricity excitation. In the inner region of the system, super-Earth planets may be survival in habitable zone (0.25-0.36 AU).
\end{abstract}

\section{INTRODUCTION}

OGLE-06-109L system is the first multiple planet system observed by microlensing technique. Table 1 lists their nominal orbital elements from Gaudi et al. (2008) [1]. The eccentricity of the outer planet $\left(e_{c}\right)$ is comparable to that of Saturn (0.01-0.09). The similarities between OGLE-06-109L system and the solar system indicate that they may have passed through similar histories during their formation stage. Several mechanisms account for the eccentricity excitation. We consider three possible scenarios for eccentricity formation of $e_{b}$ and $e_{c}$ in OGLE-06-109L system: (1) convergent migration and 3:1 meanmotion resonance (MMR) trapping; (2) planetary scattering; (3) divergent migration and the 3:1 MMR crossing.

For a planet embedded in a geometrically thin and locally isothermal disk, angular momentum exchanges between the planet and the gas disk will cause a net momentum loss on the planet, which results in a fast and so-called type I migration of the planet [2-4]. Some mechanisms are proposed to reduce the speed or even reverse the direction of migration. In this paper, we test the influence of the speed of type I migration on the distribution of habitable planets in OGLE-06-109L system. We believe that super-Earth planets can be survival in its habitable zone (HZ, 0.25-0.36 AU).

\section{MODEL AND RESULTS}

We use $N$-body simulations to test the eccentricity formation scenarios. According to our simulations in Wang, Zhao \& Zhou (2009) [5], model (1) is unlikely the useful mechanism, because the possibility of two giant planets in 3:1 MMR is low in the nominal system. Model (2) or (3) may be the reasonable mechanism for the eccentricity excitation. Model (2) favors to the system with their eccentricity evolution in the timescale of secular motion. If the eccentricities of two planets oscillate in a timescale of nearby MMR, model (3) may be the proper mechanism for the eccentricity excitation.

In order to test the influence of type I migration on the formation and final locations of planets in the HZ, we use the scenario (2) similar to Wang, Zhao \& Zhou (2009) [5], but with type I migration of embryos being considered additionally. To account for the uncertainty of the type I migration, we adopt

ae-mail: suwang@nju.edu.cn

This is an Open Access article distributed under the terms of the Creative Commons Attribution-Noncommercial License 3.0, which permits unrestricted use, distribution, and reproduction in any noncommercial medium, provided the original work is properly cited. 
Table 1. Orbital Parameters of OGLE-06-109L Planetary System.

\begin{tabular}{lccccc}
\hline Planet & $\begin{array}{c}a \\
(\mathrm{AU})\end{array}$ & $\begin{array}{c}P \\
(\text { days })\end{array}$ & $e$ & $\varpi$ & $\begin{array}{c}m \\
\left(M_{J}\right)\end{array}$ \\
\hline $\mathrm{b}$ & $2.3( \pm 0.2)$ & $1825( \pm 365)$ & $\ldots$ & $\ldots$ & $0.71( \pm 0.08)$ \\
$\mathrm{c}$ & $4.6( \pm 0.5)$ & $5100( \pm 730)$ & $0.11\left({ }_{-0.04}^{+0.17}\right)$ & $\ldots$ & $0.27( \pm 0.03)$ \\
\hline
\end{tabular}

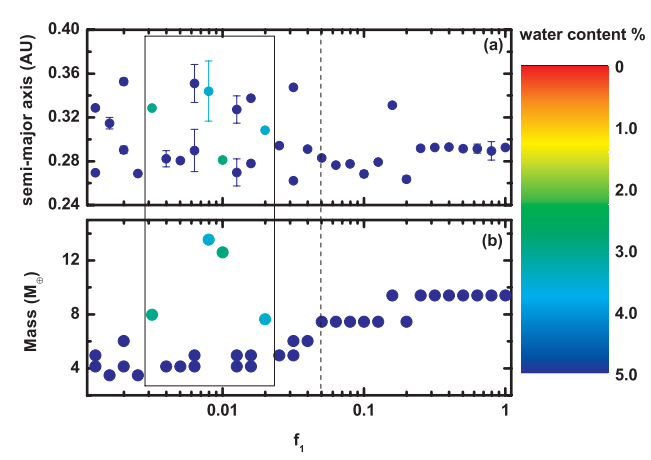

Figure 1. Distribution of habitable planets at the final stage $(t=10 \mathrm{Myr})$ of our simulations. (a) Semimajor axis distribution of habitable planets for different reduction factor $\left(f_{1}\right)$ of the type I migration. (b) Mass distribution of habitable planets for different $f_{1}$. The error bars in Panel (a) show $a(1+e)$ and $a(1-e)$ respectively. The color map represents the water content of the planets.

a speed reduction factor $f_{1}$ over the standard formula [6]. Initially 18 embryos with isolation masses ( $\mathrm{fd}=2$, two times of minimum solar nebular mass) are put in the inner region of the system [7]. Two giant planets with an additional embryo between them are also presented. A scattering will be occurred between one planet and the additional embryo at about 5 Myr, just as in Wang, Zhao \& Zhou (2009) [5], to account for the observed eccentricities of the two planets. We perform 31 runs in the same disk model but with different $f_{1}$. The results of the simulations are summarized here (for details, see Wang \& Zhou, in preparation).

Figure 1 shows that there are planets survival in the habitable zone when $f_{1}$ in the range of $[0.001,1]$. If type I migration of the embryos are fast, $f_{1}>0.05$, most of the small embryos originally in the $\mathrm{HZ}$ will run into the central star, leaving planets migrated from outside farther than $2.2 \mathrm{AU}$ with masses higher than $7 M_{\oplus}$. When $f_{1}<0.05$, embryos with masses lower than $4 M_{\oplus}$ can stay in the HZ except for $f_{1}=[0.003,0.025]$. In Figure 1, the color map shows the water distribution by mass [8]. The inner disk is water-poor, embryo within $0.5 \mathrm{AU}$ is $0.001 \%$ by mass, while embryo beyond $0.625 \mathrm{AU}$ is wet with the water content $5 \%$. Embryo between them is moderately wet with $0.1 \%$ water. If $f_{1}=[0.003,0.025]$, planets with the masses higher than $7 M_{\oplus}$ are drier than others in this region. As we assume, the water content will be decreased by $30 \%$ during each collision, these drier planets with eccentric orbits are merged from small embryos.

\section{SUMMARY}

In this paper, we study the eccentricity formation and habitable planets in the OGLE-06-109L system. Planetary scattering model or resonance crossing model can explain the eccentricity excitation in the system, these scenarios can be used for other systems. The timescale of the eccentricity evolution will decide which model is the proper one.

When $f_{1}$ is in the range of $[0.001,1]$, we believe that super-Earth planets survival in the HZ. If the mass of the habitable planet is higher than $7 M_{\oplus}$ with near-coplanar orbit, the habitable planet may 
Research, Science and Technology of Brown Dwarfs and Exoplanets

undergo migration faster than a twentieth of the standard speed. While the high mass habitable planet is in an orbit with the eccentricity about $0.08, f_{1}=[0.003,0.025]$ is the proper reduction for the type I migration. The reduction factor may be smaller than 0.05 if habitable planet is about $4 M_{\oplus}$.

This work is supported by NSFC (10925313, 10833001, 10778603), National Basic Research Program of China (2007CB814800)

\section{References}

[1] B. S. Gaudi, et al. Science, 319, 927 (2008)

[2] P. Goldreich, S. Tremaine, ApJ, 233, 857 (1979)

[3] W. R. Ward, Icarus, 126, 261 (1997)

[4] H. Tanaka, T. Takeuchi, W. R. Ward, ApJ, 565, 1257 (2002)

[5] S. Wang, G. Zhao, Ji-Lin Zhou, ApJ, 706, 772 (2009)

[6] P. Cresswell, R. P. Nelson, A \& A, 450, 833 (2006)

[7] S. Ida, D. N. C. Lin, ApJ, 604, 388 (2004)

[8] S. N. Raymond, T. Quinn, J. I. Lunine, Icarus, 168, 1 (2004) 